\title{
Tecnologias utilizadas pela enfermagem com mulheres em aleitamento materno: uma revisão integrativa
}

\author{
Technologies used by nursing with women on breastfeeding: an integrative review
}

Tecnologías utilizadas por la lactancia materna con mujeres en la lactancia materna: una revisión

integradora

Recebido: 24/09/2021 | Revisado: 30/09/2021 | Aceito: 13/10/2021 | Publicado: 16/10/2021

\author{
Denise Antunes de Azambuja Zocche \\ ORCID: https://orcid.org/0000-0003-4754-8439 \\ Universidade do Estado de Santa Catarina, Brasil \\ E-mail: denise.zocche@udesc.br \\ Andreia Cristina Dall'Agnol \\ ORCID: https://orcid.org/0000-0001-7011-9697 \\ Associação Hospitalar Lenoir Vargas Fereira, Brasil \\ E-mail: enfandreia21@gmail.com \\ Silvana dos Santos Zanotelli \\ ORCID: https://orcid.org/0000-0001-5357-0275 \\ Universidade do Estado de Santa Catarina, Brasil \\ E-mail: silvana.zanotelli@udesc.br
}

\begin{abstract}
Resumo
Este artigo teve como objetivo conhecer quais são as tecnologias que melhoram a comunicação da enfermagem com as mulheres em amamentação. A metodologia utilizada foi de uma pesquisa qualitativa com ênfase na revisão integrativa das bases de dados BVS e SCOPUS, em português, inglês e espanhol. Os estudos incluídos foram artigos originais publicados de 2006 a 2019, com os seguintes descritores: "aleitamento materno", "comunicação em saúde", "enfermagem", "mulheres" e "tecnologia”. Foram identificados 118 estudos que, após a seleção, resultaram num total de vinte e seis estudos analisados em três categorias: (1) empoderamento e autoeficácia materna para o aleitamento materno; (2) conceitos e práticas de amamentação e (3) práticas de educação em saúde na promoção do aleitamento materno. Para análise dos resultados foi utilizado à classificação de tecnologias duras, leve-duras e leves de Merhy. As tecnologias em saúde ainda são pouco utilizadas pelos enfermeiros na comunicação com mulheres em aleitamento, ainda que as tecnologias duras e leve-duras às tenham sido mencionadas em parte, pelos profissionais de saúde. Contudo, percebe-se que nos últimos cincos anos houve aumento na produção de estudos sobre a comunicação dos enfermeiros com mulheres que amamentam. Destaca-se entre elas, o uso de tecnologias educativas para o empoderamento e autoeficácia das mulheres na amamentação.
\end{abstract}

Palavras-chave: Aleitamento materno; Comunicação em saúde; Enfermagem; Tecnologia; Mulheres.

\begin{abstract}
This article aimed to know which technologies improve nursing communication with breastfeeding women. The methodology used was a qualitative research with emphasis on the integrative review of the VHL and SCOPUS databases, in Portuguese, English and Spanish, The included studies were original articles published from 2006 to 2019, with the following descriptors: "breastfeeding"," health_communication", "nursing", "women" and "technology". A total of 118 studies were identified, resulting in a total of twenty- six studies analyzed in three categories: (1) empowerment and maternal self-efficacy for breastfeeding; (2) concepts and practices of breastfeeding and(3) health education practices in the promotion of breastfeeding. For the analysis of the results, the classification of Hard, light-hard and light Merhy technologies. Health technologies are still little used by nurses in communication with breastfeeding women even though hard and light-to-hard technologies have been mentioned in part by health professionals. However, it is noticed that in the last five years there has been an increase in the production of studies on the communication of nurses with breastfeeding women. Among them, the use of educational technologies for the empowerment and self-efficacy of women in breastfeeding stands out.
\end{abstract}

Keywords: Breastfeeding; Health communication; Nursing; Technology; Women.

\section{Resumen}

Este artículo tuvo como objetivo conocer qué tecnologías mejoran la comunicación de la lactancia con las mujeres que amamantan. La metodología utilizada fue una investigación qualitativas con énfasis en la revisión integradora de las bases de datos BVS y SCOPUS, en portugués, inglés y español. Los estudios incluidos fueron artículos originales publicados entre 2006 y 2019, con los siguientes descriptores: "lactancia materna", "comunicación en salud", "enfermería", "mujeres" y "tecnología". Se identificaron 118 estudios que, después de la selección, dieron como 
resultado un total de veintiséis estudios analizados en tres categorías: (1) empoderamiento y autoeficacia materna para la lactancia materna; (2) conceptos y prácticas de lactancia materna y (3) prácticas de educación para la salud en la promoción de la lactancia materna. Para el análisis de los resultados, se utilizaron las tecnologías hard, light-hard y light de Merhy para analizar los resultados. Las tecnologías de la salud todavía son poco utilizadas por las enfermeras en la comunicación con las mujeres que amamantan, aunque las tecnologías duras y ligeras han sido mencionadas en parte por los profesionales de la salud. Sin embargo, se observa que en los últimos cinco años ha habido un aumento en la producción de estudios sobre la comunicación de las enfermeras con las mujeres lactantes. Entre ellas, destaca el uso de tecnologías educativas para el empoderamiento y autoeficacia de las mujeres en la lactancia materna.

Palabras clave: Lactancia materna; Comunicación en salud; Enfermería; Tecnología; Mujeres.

\section{Introdução}

O aleitamento materno é a mais sábia estratégia natural de vínculo, afeto, proteção e nutrição para a criança. Constitui uma intervenção, sensível, econômica e eficaz para a redução da morbimortalidade infantil, além de permitir um impacto positivo na promoção da saúde integral do binômio mãe-filho (Costa, et al., 2013, Joventino, et al., 2011).

O leite materno é uma fonte única de nutrientes, é considerado o alimento ideal para um crescimento adequado nos primeiros seis meses de vida e deve ser ofertado de forma exclusiva, sem a necessidade de complementação. Dessa forma, a mãe deve ser incentivada e orientada quanto à manutenção do Aleitamento Materno (AM) e principalmente do Aleitamento Materno Exclusivo (AME) (Costa, et al., 2013, Marinho, et al., 2015, Marques, et al., 2004, Dias, et al., 2016).

Nesse sentido, o enfermeiro, busca pelo sucesso da amamentação na sua prática clínica, e vem, cada vez mais, fazendo uso de tecnologias como recurso para auxiliar na assistência a essa clientela, buscando melhores resultados para o desenvolvimento da adesão e manutenção do aleitamento materno (Marinho, et al., 2015, Silva, et al., 2014).

$\mathrm{O}$ apoio às mulheres que vivenciam o processo de amamentar deve ser permanente durante todo o período da amamentação, sendo fundamental que o enfermeiro esteja atualizado em seus conhecimentos e condutas para desenvolver atividades de forma eficiente, para melhor atender a clientela. Nesse contexto, torna-se necessário o uso de tecnologias adequadas para cada família, a fim de que o cuidado por ele prestado seja considerado eficaz e de qualidade (Joventino, et al., 2011, Marinho, et al., 2015, Prates, et al., 2015, Silva, et al., 2019).

Percebe-se que o uso de tecnologias contribui para a educação e promoção da saúde da população ao permitir a organização ou a utilização de recursos educacionais e tecnológicos, visando simplificar o trabalho e melhorar o ensinoaprendizagem (Silva, et al., 2019, Oriá, et al., 2018).

Os tipos de tecnologia, as quais o profissional de enfermagem pode valer-se podem ser classificadas em três: tecnologia dura (instrumentos, normas e equipamentos tecnológicos); tecnologia leve-dura (teorias, modelos de cuidado, processo de enfermagem) e tecnologias leves (vinculo, gestão de serviços e acolhimento) (Merhy, 2002).

Considerando os aspectos mencionados, o presente estudo objetivou a realizar uma revisão integrativa da literatura sobre as tecnologias que melhoram a comunicação da enfermagem com as mulheres em amamentação. Use o parágrafo como modelo.

\section{Metodologia}

Para o alcance do objetivo proposto neste estudo selecionou-se como método de pesquisa a revisão integrativa. Este tipo de estudo busca a identificação e análise das evidências existentes de práticas de saúde, abrangendo a literatura teórica e empírica (Ribeiro, et al., 2012).

O objetivo da pesquisa bibliográfica e/ou integrativa é o de conhecer e analisar as principais contribuições teóricas existentes sobre um determinado tema ou problema, tornando-se um instrumento indispensável para qualquer tipo de pesquisa, sendo utilizada para ampliar o grau de conhecimento em uma determinada área e capacitando para dominar o conhecimento disponível (Köche, 2011). 
As etapas percorridas foram: 1: definição da equipe responsável; 2: identificação da questão de pesquisa e descritores; 3: avaliação do protocolo; 4: seleção e extração dos estudos; 5: validação da seleção dos estudos; 6: avaliação dos estudos incluídos; 7: Análise e interpretação dos resultados da revisão; 8: apresentação dos resultados; 9: discussão dos resultados e 10: considerações finais.

Estabeleceu-se a seguinte pergunta norteadora: "Que tecnologias melhoram a comunicação da enfermagem com as mulheres em amamentação?".

Os critérios de inclusão adotados pelo presente estudo foram: publicações disponíveis eletronicamente, artigos completos, disponíveis no período de 2006 a 2019, após o $1^{\text {o }}$ Seminário Nacional de Políticas Públicas em Aleitamento Materno; estar divulgado em inglês, espanhol e português, disponíveis nas bases Biblioteca Virtual em Saúde (BVS) e SCOPUS $^{\circledR}$. Foram excluídos as teses, dissertações, opinião e relatos de experiências, artigos não disponíveis eletronicamente, bem como estudos que não abordassem a temática do objetivo do estudo.

Utilizou-se a terminologia em saúde consultada nos Descritores em Ciências da Saúde (DeCS) pelo qual se identificaram os descritores, sendo: aleitamento materno (breast feeding) (lactancia materna); comunicação em saúde (health communication) (comunicación em salud); enfermagem (nursing) (enfermeira); mulheres (women) (mujeres) e tecnologia (technology) (tecnologia). As bases de dados utilizadas permitiram realizar uma busca avançada com três descritores ao mesmo tempo, utilizando o operador booleano "AND” como fator de ligação.

A busca ocorreu no período de agosto a setembro de 2019, por meio da realização de quatro cruzamentos: "aleitamento materno AND comunicação em saúde AND tecnologia", "aleitamento materno AND enfermagem AND tecnologia", "aleitamento materno AND mulheres AND tecnologia", "aleitamento materno AND enfermagem AND comunicação em saúde" que resultou na seleção de 118 artigos. Esta seleção compreendeu a leitura dos títulos e resumos a fim de selecionar aqueles que seriam incluídos, pois contemplavam a pergunta norteadora. Nesta etapa, identificou-se 16 (14\%) estudos duplicados, 67 (57\%) não atenderam temática do estudo, 04 (3\%) relatos de experiências, 01 (1\%) tese, 04 (3\%) não atenderam a pergunta norteadora do estudo, e que foram excluídos. Ao final somente foram incluídos 26 (22\%) artigos, conforme a Figura 1 abaixo: 
Figura 1. Fluxo de seleção dos artigos nas bases científicas BVS e SCOPUS.

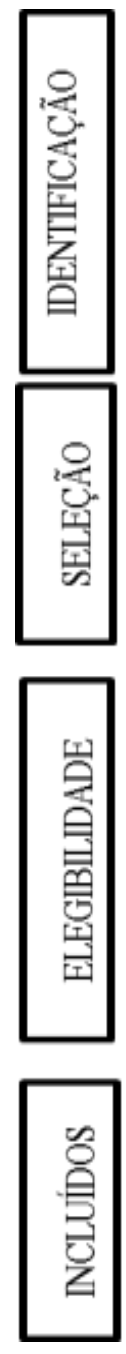

118 Artigos identificados na busca nas bases BVS e SCOPUS

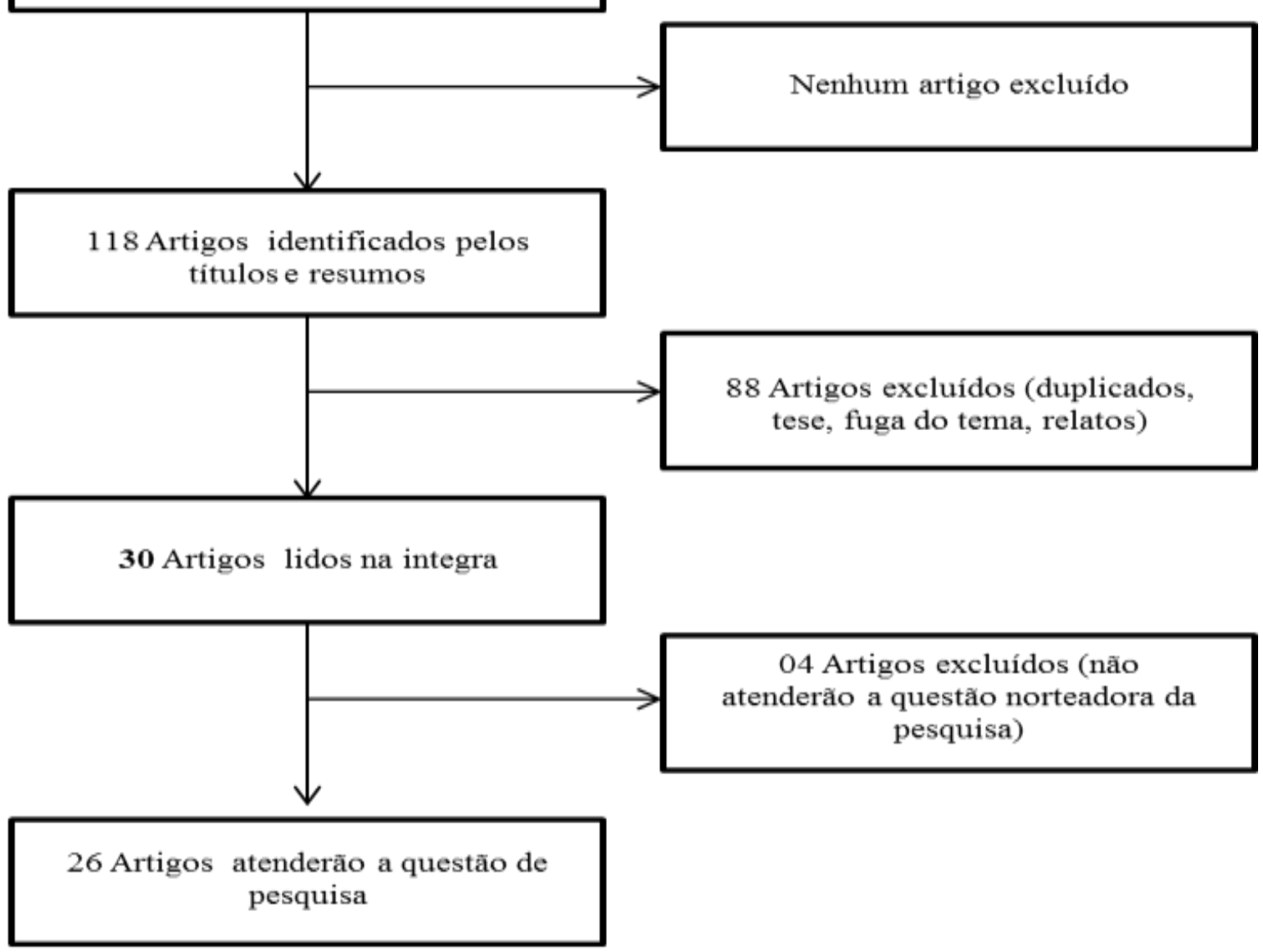

Fonte: Autores (2020).

Cabe destacar que, esta seleção foi validada por dois especialistas na área, a fim de garantir a adequação entre os descritores, e a pergunta de pesquisa. Para tanto se utilizou o protocolo de revisão integrativa desenvolvido no Mestrado Profissional em Enfermagem na Atenção Primária à Saúde (MPEAPS) da Universidade do Estado de Santa Catarina (UDESC) (Zocche, et al., 2020). Os artigos foram analisados por meio de tradução, leitura e releitura rigorosas a fim de extrair as ideias principais e sua relação com a pergunta de pesquisa. Para tanto foi utilizado um instrumento de avaliação e análise dos dados extraídos (Zocche, et al., 2020). Além disso, foram construídas tabelas em Microsoft ${ }^{\circledR}$ Word contendo informações de cada artigo, sobre origem, ano, tipo de estudo, área de especialidade da enfermagem, nível de evidência, para identificar padrões recorrentes, falhas nos estudos que poderiam afetar os resultados. Para avaliar o nível de evidência foi utilizada a pirâmide de nível de evidência e a classificação de Oxford Center for Evidence-Based Medicine (Montagna, et al., 2020, Murad, et al., 2016).

\section{Resultados}

\subsection{Caracterização dos estudos selecionados quanto ao período e origem}

Por meio da análise dos 26 artigos selecionados, verificou-se que a maioria dos estudos eram do Brasil (16 - 62\%), sendo em sua maioria da região Nordeste (9-56\%), seguido pela região Sul (4 - 25\%) e a região Sudeste (3 - 19\%).Nas publicações internacionais (10 -38\%), destaca-se a América do Norte (4 -40\%) seguido da Oceania (3 - 30\%) e Europa (3 - 
$30 \%$ ). Observou-se um aumento significativo na publicação sobre a temática do aleitamento materno e tecnologias (21 - $81 \%)$, nos últimos 10 anos, sendo que o ano de 2018 teve o maior número (5 - 19\%).

\subsection{O uso das tecnologias duras, leve-duras e leves pela enfermagem na amamentação}

Quanto aos tipos de tecnologias presentes, o estudo revelou que as tecnologias utilizadas na comunicação em enfermagem com mulheres durante a amamentação são: tecnologia leve (4 - 15\%), leve-dura (10 - 39\%), e dura (12 - 46\%) e são aplicadas pela enfermagem preferencialmente através da tecnologia educacional (18 - 69\%) seguido da tecnologia assistencial (8-31\%) (Quadro 1).

Quadro 1. Categorização dos estudos.

\begin{tabular}{|c|c|c|c|c|}
\hline $\begin{array}{c}\text { Caracterização (tipo, método, nível evidência, } \\
\text { área do conhecimento, subárea da } \\
\text { enfermagem, país de origem) }\end{array}$ & Abordagem & $\begin{array}{l}\text { Grau de adesão à } \\
\text { pergunta de Pesquisa } \\
\text { (forte, média, fraca) }\end{array}$ & $\begin{array}{c}\text { Tipo de } \\
\text { tecnologia }\end{array}$ & $\begin{array}{l}\text { Aplicação da } \\
\text { tecnologia na } \\
\text { enfermagem }\end{array}$ \\
\hline $\begin{array}{l}\text { A-1: Metodológica, qualitativo, nível 4, saúde e } \\
\text { educação, tecnologia educacional, país Brasil. }\end{array}$ & Qualitativa & Forte & Leve-dura & Educacional \\
\hline $\begin{array}{l}\text { A-2: Revisão sistemática, nível 1A, enfermagem } \\
\text { Atenção Saúde Pública, saúde pública, Brasil. }\end{array}$ & Qualitativa & Forte & Dura & Educacional \\
\hline $\begin{array}{l}\text { A-3: Intervenção, controlado e randomizado, } \\
\text { misto, nível 1B, saúde e educação, tecnologia } \\
\text { educacional, Brasil. }\end{array}$ & Misto & Forte & Dura & Educacional \\
\hline $\begin{array}{l}\text { A-4: Ensaio clínico randomizado, qualitativa, } \\
\text { nível 1B, saúde e educação, tecnologia } \\
\text { educacional, Brasil. }\end{array}$ & Qualitativa & Média & Dura & Educacional \\
\hline $\begin{array}{l}\text { A-5: Metodológica, qualitativo, nível 4, saúde e } \\
\text { educação, tecnologia educacional, Brasil. }\end{array}$ & Qualitativa & Média & Leve-dura & Educacional \\
\hline $\begin{array}{l}\text { A-6: Metodológica, qualitativa, nível } 4, \\
\text { enfermagem atenção } \\
\text { pública, Brasil. }\end{array}$ & Qualitativa & Forte & Leve-dura & Educacional \\
\hline 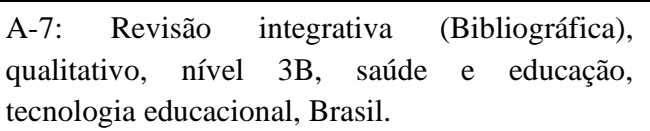 & Qualitativa & Média & Leve & Educacional \\
\hline $\begin{array}{l}\text { A-8: Descritiva, quantitativa, nível } 4 \text {, } \\
\text { enfermagem atenção saúde pública, saúde } \\
\text { pública, Brasil. }\end{array}$ & Quantitativa & Forte & Leve-dura & Assistencial \\
\hline $\begin{array}{l}\text { A9- Experimental, quantitativo, nível 3A, saúde e } \\
\text { educação, tecnologia educacional, Brasil. }\end{array}$ & Quantitativa & Forte & Dura & Educacional \\
\hline $\begin{array}{l}\text { A-10: Descritiva, qualitativa, nível } 4, \\
\text { enfermagem atenção saúde pública, enfermagem } \\
\text { obstétrica, Brasil. }\end{array}$ & Qualitativa & Fraca & Leve-dura & Educacional \\
\hline $\begin{array}{l}\text { A-11: Descritivo, comparativo, misto, nível } 4 \text {, } \\
\text { enfermagem atenção saúde pública, obstétrica, } \\
\text { Portugal. }\end{array}$ & Misto & Média & Leve-dura & Assistencial \\
\hline $\begin{array}{l}\text { A-12: Fenomenológica, qualitativo, nível } 5 \text {, } \\
\text { enfermagem atenção saúde pública, enfermagem } \\
\text { obstétrica, Brasil. }\end{array}$ & Qualitativa & Fraca & Leve & Assistencial \\
\hline $\begin{array}{l}\text { A-13: Metodológica, quantitativo, nível 4, saúde } \\
\text { e educação, tecnologia educacional, EUA. }\end{array}$ & Quantitativa & Forte & Dura & Educacional \\
\hline $\begin{array}{l}\text { A-14: Sistemática e exploratória, qualitativo, } \\
\text { nível 4, saúde e educação, tecnologia } \\
\text { educacional, Canada. }\end{array}$ & Qualitativa & Forte & Dura & Educacional \\
\hline
\end{tabular}




\begin{tabular}{|c|c|c|c|c|}
\hline $\begin{array}{l}\text { A-15: Longitudinal prospectiva, observacional e } \\
\text { analítica, qualitativo, nível 4, enfermagem } \\
\text { atenção saúde pública, enfermagem obstétrica, } \\
\text { Brasil. }\end{array}$ & Qualitativa & Média & Leve-dura & Educacional \\
\hline $\begin{array}{l}\text { A-16: Ensaio clínico controlado e randomizado, } \\
\text { qualitativo, nível 1B, saúde e educação, } \\
\text { tecnologia educacional, Brasil. }\end{array}$ & Qualitativa & Forte & Dura & Educacional \\
\hline $\begin{array}{l}\text { A-17: Metodológica, qualitativo, nível 4, área de } \\
\text { conhecimento saúde e educação, subárea } \\
\text { tecnologia educacional, país Portugal. }\end{array}$ & Qualitativa & Forte & Dura & Educacional \\
\hline $\begin{array}{l}\text { A-18: Descritiva, exploratória, qualitativo, nível } \\
\text { 5, enfermagem atenção saúde pública, } \\
\text { enfermagem obstétrica, Brasil. }\end{array}$ & Qualitativa & Média & Leve & Assistencial \\
\hline $\begin{array}{l}\text { A-19: Observacional, prospectivo e não } \\
\text { comparativo, transversal, quantitativo, nível } 5 \text {, } \\
\text { enfermagem atenção saúde pública, enfermagem } \\
\text { obstétrica, Brasil. }\end{array}$ & Quantitativa & Fraca & Dura & Assistencial \\
\hline $\begin{array}{l}\text { A-20: Coorte prospectivo, quantitativo, nível 2B, } \\
\text { enfermagem atenção saúde pública, saúde } \\
\text { pública, Austrália. }\end{array}$ & Quantitativa & Forte & Dura & Educacional \\
\hline $\begin{array}{l}\text { A-21: Relato experiência, qualitativo, nível } 5 \text {, } \\
\text { enfermagem atenção saúde pública, enfermagem } \\
\text { obstétrica, Itália. }\end{array}$ & Qualitativa & Fraca & Dura & Assistencial \\
\hline $\begin{array}{l}\text { A-22: Exploratória (análise de discurso), } \\
\text { qualitativa, nível 5, enfermagem atenção saúde } \\
\text { pública, enfermagem obstétrica, Austrália. }\end{array}$ & Qualitativa & Fraca & Leve-dura & Assistencial \\
\hline $\begin{array}{l}\text { A-23: Retrospectivo, quantitativo, nível 5, } \\
\text { enfermagem atenção saúde pública, saúde } \\
\text { pública, EUA. }\end{array}$ & Quantitativa & Fraca & Leve-dura & Educacional \\
\hline $\begin{array}{l}\text { A-24: Descritiva, reflexiva, qualitativo, nível } 5 \text {, } \\
\text { enfermagem atenção saúde pública, saúde } \\
\text { pública, Oceania. }\end{array}$ & Qualitativa & Média & Leve-dura & Educacional \\
\hline $\begin{array}{l}\text { A-25: Fenomenológica, qualitativo, nível } 4 \text {, } \\
\text { enfermagem atenção saúde pública, saúde } \\
\text { pública, USA. }\end{array}$ & Qualitativa & Média & Leve & Educacional \\
\hline $\begin{array}{l}\text { A-26: Ensaio clínico randomizado controlado, } \\
\text { quantitativo, nível } 1 \mathrm{~B} \text {, enfermagem atenção saúde } \\
\text { pública, enfermagem obstétrica, Brasil. }\end{array}$ & Quantitativa & Forte & Dura & Educacional \\
\hline
\end{tabular}

Fonte: Autores (2020).

A tecnologia educacional leve-dura foi a que mais se destacou, seguida pela tecnologia assistencial dura. No contexto da amamentação, a tecnologia representa um conjunto de conhecimentos e práticas que se relacionam para produzir saúde. Neste processo, o enfermeiro não pode ser substituído neste processo pela tecnologia dura ou leve-dura, mas sim ser integrado, pois a interação entre o profissional de saúde e a mulher que amamenta fortalece a prática de amamentação (Silva, et al., 2014). As tecnologias leve-duras apresentadas nos artigos do estudo são: literatura de cordel, instrumentos de validação desta literatura de cordel, instrumentos de pesquisas, diagnósticos de enfermagem para banco de leite humano (BLH), protocolo de observação de mamas, escala de autoeficácia materna em amamentar, áudios, seminário, utilização de teorias para análise de entrevistas. Essas são formas de tecnologias leve-duras utilizadas pela enfermagem para melhorar a comunicação com as mulheres em amamentação, bem como meios para aprimorar e qualificar a atuação profissional. 
Após análise dos estudos encontrados, foram criadas três categorias que utilizam as tecnologias para promover, proteger e apoiar o aleitamento materno: Empoderamento e autoeficácia materna para o aleitamento materno; Conceitos e práticas de amamentação; e Práticas de educação em saúde na promoção do aleitamento materno.

\section{Discussão}

A enfermagem vem utilizando tecnologias para mediar o cuidado prestado à mulher no processo de amamentação, bem como sua rede de apoio. O avanço da ciência e tecnologia tem proporcionado uma variedade de intervenções disponíveis para que o profissional da saúde possa melhorar sua comunicação com essas mulheres e influenciar diretamente na duração do aleitamento materno.

\subsection{Empoderamento e autoeficácia materna para o aleitamento materno}

A autoeficácia materna é a crença na habilidade pessoal de executar com êxito determinadas tarefas ou comportamentos para alcançar resultados desejáveis, sendo um fator modificável principalmente por meio da educação da saúde (Dodt, et al., 2015, Rodrigues, et al., 2017). A confiança materna em amamentar está relacionada à percepção da mulher sobre a sua capacidade para amamentar seu filho, significando que as mães precisam acreditar que têm conhecimentos e habilidades para realizar a amamentação com êxito, tornando essa prática bem sucedida (Rodrigues, et al., 2017, Conde, et al., 2017).

Este estudo revelou que a autoeficácia em amamentar é uma variável que pode ser modificada por meio de intervenções educativas e apoio social, nos artigos A3, A9, A13, A14 e A18 identificou-se que a maioria da tecnologia aplicada nesta categoria era a tecnologia educacional dura a qual permite fortalecer a comunicação com a mulher em amamentação e aumentar a sua confiança neste período, utilizando como alicerce as quatro fontes de crenças imbricadas na construção da confiança da mulher em amamentar, como: a experiência direta ou anterior com a amamentação, as experiências vicárias, a persuasão verbal e as reações físicas e emocionais permitindo o aprendizado e adaptação para mãe, filho e família (Rodrigues, et al., 2017, Conde, et al., 2017, Javorski, et al., 2018).

A utilização por parte dos profissionais de saúde das tecnologias educacionais e assistenciais de forma precoce apontadas nos artigos A3, A9, A12 e A16 durante o período do pré-natal e no puerpério através de abordagens individuais ou grupais utilizando tecnologias duras como álbum seriado e flipt chart, bem como a procura por parte das mulheres por assuntos relacionados com amamentação nas redes sociais (A13 e A14), grupos no Facebook®, Instagram® promovem e melhoram a autoeficácia materna em amamentar, refletem no aumento do período de aleitamento materno exclusivo(Dodt, et al., 2015, Rodrigues, et al., 2017, Conde, et al., 2017, Javorski, et al., 2018, Monteiro, et al., 2006, Robinson, et al., 2019, Marcon, et al., 2019).

\subsection{Conceitos e práticas de amamentação}

Nos últimos anos tem sido evidenciado um interesse nas ações e programas em saúde pública voltada a atenção e cuidado materno-infantil, dando ênfase à prática da amamentação, sendo considerada uma estratégia econômica e eficaz na redução da morbimortalidade materno-infantil (Oriá, et al., 2018). É importante ressaltar ainda o apoio da figura paterna e da família na prática de amamentação que proporcionam aumento nas taxas e prolonga a duração do aleitamento materno exclusivo, surgindo a importância do profissional da saúde, especialmente o enfermeiro com habilidade de comunicação auxiliar com êxito neste momento (Oliveira, et al., 2017).

Os profissionais da saúde devem estar conscientes e preparados para prestar uma assistência eficaz, solidária, integral e contextualizada a mulher e família neste período. É essencial respeitar saberes, experiências e histórias de vida de cada 
mulher e sua família, assim ajuda-las a superar medos, dificuldades e inseguranças que se apresentam no período de amamentação (Silva, et al., 2014, Galvão, 2011, Oliveira, et al., 2014). Sobre os fatores que podem dificultar a manutenção da amamentação, destacam-se os estudos A4, A8, A10, A11, A17 e A20 que apontam como um meio principal a posição e pega inadequada ao seio materno refletindo em ingurgitamento mamário, fissuras mamilares, dor, gerando medos, ansiedades e diminuição na autoconfiança materna proporcionando a introdução alimentar precoce e inadequada para a idade da criança, uso de mamadeira e bicos artificiais e assim o desmame precoce (Silva, et al., 2014, Galvão, 2011, Oliveira, et al., 2014, Martins, et al., 2018, Crespo, et al., 2019, Hammarberg, et al., 2010).

Para obter sucesso e efetivação da amamentação na prática clínica, identificou-se o uso de tecnologias como recurso para auxiliar na assistência a essa clientela, buscando melhores resultados na confiança, adesão e manutenção do aleitamento materno. A prática educativa do enfermeiro deve valorizar o uso de estratégias e tecnologias que contribuam para oferecer apoio, suporte e orientação necessários para a prática da amamentação (Silva, et al., 2014, Oriá, et al., 2018).

Nos estudos A1, A2 e A5 a tecnologia permeia todo o processo de trabalho em saúde, sendo um grande aliado no desenvolvimento de orientações, capacitações e intervenções pelo profissional enfermeiro em relação à prática de amamentação. Esses estudos reforçam que a enfermagem, diante da busca pelo sucesso na amamentação vem fazendo uso de tecnologias que contribui para oferecer apoio, suporte e orientação necessários para a prática da amamentação abordando a composição do leite materno, mitos e tabus, vantagens da amamentação para a criança, mãe, família e melhorando a comunicação em saúde. Comunicação em saúde é construída da capacidade de transmitir e receber informação com base no contexto ambiental (Silva, et al., 2014, Oriá, et al., 2018, Oliveira, et al., 2017, Oliveira, et al., 2009).

\subsection{Práticas de educação em saúde na promoção do aleitamento materno}

A estratégia de educação em saúde voltada a promoção em aleitamento materno, vem sendo cada vez mais discutidas no âmbito da saúde coletiva e hospitalar, visando à redução da morbimortalidade materna e infantil além de proporcionar a mais sábia estratégia natural de vínculo, afeto, proteção e nutrição para a criança (Joventino, et al., 2011).

O desenvolvimento e uso de estratégias de educação em saúde constituem-se em um componente essencial para o trabalho da enfermagem na área da saúde comunitária, podendo estar voltada para a promoção, manutenção e adaptação a alguma situação ou prática (Joventino, et al., 2011). A tecnologia empregada diariamente nas funções executadas pelo enfermeiro compreendendo um conjunto de saberes e fazeres relacionado ao cuidado, sendo considerado ao mesmo tempo processo e produto, contribuindo para a estratégia de educação e promoção da saúde (Joventino, et al., 2011).

Portanto, é notório o papel que o enfermeiro desempenha na função de educador e promotor da saúde principalmente na temática de aleitamento materno para promoção, manutenção e recuperação da saúde, executando com criatividade e competência a arte do cuidar fazendo uso de diversas tecnologias (Joventino, et al., 2011, Oriá, et al., 2018, Oliveira, et al., 2013). Os estudos A6, A7 e A26 reforçam que, a intervenção educativa com auxilio de tecnologias em curto e longo prazo é capaz de elevar a autoeficácia materna em amamentar e, a duração do aleitamento materno, mas não a sua exclusividade (Joventino, et al., 2011, Oliveira, et al., 2013, Chaves, et al., 2019). Estes estudos ainda apontam que o enfermeiro deve utilizar os conhecimentos prévios dsa gestantes e familiares sobre aleitamento materno. Assim o enfermeiro pode fazer uso da tecnologia mais adequada para cada mãe, bebê e família, a fim de qualificar a promoção do aleitamento materno.

Reforçando essa ideia, A23 e A25 apontam para a importância do apoio a amamentação englobando uma visão ampla relacionada com a cultura e origem tanto materna quanto do profissional voltada ao diálogo, informação e capacitação que empoderam as mães para reconhecer e interpretar as respostas de seus recém-nascidos frente ao aleitamento materno (Noble, et al., 2009, Grassley, et al., 2008). 


\section{Conclusão}

O estudo revelou que a maioria das tecnologias empregada na comunicação com as mulheres em aleitamento materno vem sendo a tecnologia educativa seguida da assistencial.Com relação aos tipos, o uso as tecnologias duras e leve-duras (álbum seriado, flipt chart, jogo de tabuleiro, telefone, Facebook®, Instagram®, cordel, seminário, escala de autoeficácia, instrumentos de pesquisas, entrevistas) são as mais utilizadas seguida da tecnologia leve (observação de vínculo) proporcionando a promoção, proteção e apoio ao aleitamento materno em várias dimensões de atuação do profissional enfermeiro. Destaca-se que as tecnologias educacionais duras, vem aumentando entre os profissionais de enfermagem devido à crescente demanda de cuidado com as mulheres em amamentação. Neste sentido, se faz necessário que sejam desenvolvidos mais estudos sobre o impacto das tecnologias, leve, leve-duras ou duras na prática da amamentação bem como no processo de trabalho da enfermagem e suas contribuições para a melhoria da comunicação entre enfermeiros e mulheres que amamentam.

Este estudo contribui para a melhoria na assistência do profissional de enfermagem na prática e empoderamento em relação à promoção do aleitamento materno.

\section{Referências}

Chaves, A. F. L., Ximenes, L. B., Rodrigues, D. P., Vasconcelos, C. T. M., Monteiro, J. C. D. S., \& Oriá, M. O. B. (2019). Intervenção telefônica na promoção da autoeficácia, duração e exclusividade do aleitamento materno: estudo experimental randomizado controlado. Revista Latino-Americana de Enfermagem, 27, 1-9, from https://www.scielo.br/pdf/rlae/v27/1518-8345-rlae-27-e3140.pdf..

Conde, R. G., Guimarães, C. M. D. S., Gomes-Sponholz, F. A., Oriá, M. O. B., \& Monteiro, J. C. D. S. (2017). Autoeficácia na amamentação e duração do aleitamento materno exclusivo entre mães adolescentes. Acta Paulista de Enfermagem, 30, 383-389, from https://www.scielo.br/pdf/ape/v30n4/0103-2100-ape30-04-0383.pdf.

Costa, L. K. O., Queiroz, L. L. C., da Silva Queiroz, R. C. C., Ribeiro, T. S. F., \& Fonseca, M. D. S. S. (2013). Importância do aleitamento materno exclusivo: uma revisão sistemática da literatura. Revista de Ciências da http://www.periodicoseletronicos.ufma.br/index.php/rcisaude/article/view/1920/2834.

Crespo, N. C. T., Santana, R. F., Alves, V. H., Pereira, A. V., Marchiori, G. R. S., \& Rodrigues, D. P. (2019). Diagnósticos de enfermagem de mulheres nutrizes atendidas no Banco de leite humano. Enfermagem em Foco, 10(1),12-17, http://revista.cofen.gov.br/index.php/enfermagem/article/view/1396/486

Dias, R. B., Boery, R. N. S. D. O., \& Vilela, A. B. A. (2016). Conhecimento de enfermeiras e estratégias de incentivo da participação familiar na amamentação. Ciência \& Saúde Coletiva, 21, 2527-2536, https://www.scielosp.org/article/csc/2016.v21n8/2527-2536/pt/.

Dodt, R. C. M., Joventino, E. S., Aquino, P. S., Almeida, P. C., \& Ximenes, L. B. (2015). Estudo experimental de uma intervenção educativa para promover a autoeficácia materna na amamentação. Revista latino-americana de enfermagem, 23, 725-732, https://www.scielo.br/pdf/rlae/v23n4/pt_0104-1169-rlae-23-0400725.pdf.

Galdino Y L S, Moreira T M M, Cestari V R F.(2018). Construção e validação educativa: trabalhando inovações tecnológicas. EdUECE.

Galvão, D. G. (2011). Formação em aleitamento materno e suas repercussões na prática clínica. Revista Brasileira de Enfermagem,64, 308-314, https://www.redalyc.org/pdf/2670/267019461014.pdf.

Grassley, J. S., \& Nelms, T. P. (2008). The breast-feeding conversation: a philosophic exploration of support. Advances in Nursing Science, 31(4), E55-E66, https://journals.lww.com/advancesinnursingscience/Abstract/2008/10000/The_Breast_feeding_Conversation_A_Philosophic.14.aspx.

Hammarberg, K., Fisher, J. R. W., Wynter, K. H., \& Rowe, H. J. (2011). Breastfeeding after assisted conception: a prospective cohort study. Acta paediatrica, 100(4), 529-533, https://onlinelibrary.wiley.com/doi/epdf/10.1111/j.1651-2227.2010.02095.x.

Javorski, M., Rodrigues, A. J., Dodt, R. C. M., Almeida, P. C. D., Leal, L. P., \& Ximenes, L. B. (2018). Efeitos de uma tecnologia educativa na autoeficácia para amamentar e na prática do aleitamento materno exclusivo. Revista da Escola de Enfermagem da USP, 52, 1-8, https://www.scielo.br/pdf/reeusp/v52/1980-220X-reeusp-52-e03329.pdf.

Joventino, E. S., Dodt, R. C. M., Araujo, T. L., Cardoso, M. V. L. M. L., Silva, V. M. D., \& Ximenes, L. B. (2011). Tecnologias de enfermagem para promoção do aleitamento materno: revisão integrativa da literatura. Revista Gaúcha de Enfermagem,32, 178-184, https://www.scielo.br/scielo.php?pid=S1983-14472011000100023\&script=sci_arttext.

Köche, J. C. (2011). Fundamentos de metodologia científica: teoria da ciência e iniciação à pesquisa. Vozes.

Marcon, A. R., Bieber, M., \& Azad, M. B. (2019). Protecting, promoting, and supporting breastfeeding on Instagram. Maternal \& child nutrition, 15(1), 1-12, from https://onlinelibrary.wiley.com/doi/epdf/10.1111/mcn.12658.

Marinho, M dos S., de Andrade, E. N., \& de Vilhena Abrão, A. C. F. (2015). A atuação do (a) enfermeiro (a) na promoção, incentivo e apoio ao aleitamento materno: revisão bibliográfica. Revista Enfermagem Contemporânea, 4(2), 189-198, https://www5.bahiana.edu.br/index.php/enfermagem/article/view/598.

Marques, R. F., Lopez, F. A., \& Braga, J. A. (2004). O crescimento de crianças alimentadas com leite materno exclusivo nos primeiros 6 meses de vida. Jornal de pediatria, 80, 99-105, https://www.scielo.br/scielo.php?pid=S0021-75572004000200005\&script=sci_arttext. 
Martins, F. D. P., Leal, L. P., Linhares, F. M. P., Santos, A. H. D. S., Leite, G. D. O., \& Pontes, C. M. (2018). Efeito de tecnologia educacional jogo de tabuleiro no conhecimento de escolares sobre aleitamento materno1.Revista Latino-Americana de Enfermagem, 26, 1-12, https://www.scielo.br/pdf/rlae/v26/pt_0104-1169-rlae-26-e3049.pdf.

Merhy E E.(2002). Saúde: cartografia do trabalho vivo em ato. Hucitec.

Montagna, E., Zaia, V., \& Laporta, G. Z. (2020). Adoção de protocolos para aprimoramento da qualidade da pesquisa médica. Einstein, 18,1-4. https://journal.einstein.br/wp-content/uploads/articles_xml/2317-6385-eins-18-eED5316/2317-6385-eins-18-eED5316-pt.pdf.

Monteiro, J. C. D. S., Gomes, F. A., \& Nakano, A. M. S. (2006). Percepção das mulheres acerca do contato precoce e da amamentação em sala de parto. Acta Paulista de Enfermagem, 19, 427-432, https://www.scielo.br/pdf/ape/v19n4/v19n4a10.pdf.

Murad, MH, Asi, N., Alsawas, M., \& Alahdab, F. (2016). Nova pirâmide de evidências. BMJ Evidence-Based Medicine,21 (4), 125-127. https://ebm.bmj.com/content/ebmed/21/4/125.full.pdf.

Noble, L. M., Noble, A., \& Hand, I. L. (2009). Cultural competence of healthcare professionals caring for breastfeeding mothers in urban areas. Breastfeeding Medicine, 4(4), 221-224, https://www.liebertpub.com/doi/abs/10.1089/bfm.2009.0020.

Oliveira, P. M. P. D., Carvalho, A. L. R. F. D., \& Pagliuca, L. M. F. (2014). Adaptação cultural de tecnologia educativa em saúde: literatura de cordel com enfoque na amamentação1. Texto \& Contexto-Enfermagem, 23, 134-141, https://www.scielo.br/pdf/tce/v23n1/pt_0104-0707-tce-23-01-00134.pdf

Oliveira, P. M. P. D., Pagliuca, L. M. F., Cezario, K. G., Almeida, P. C. D., \& Beserra, G. D. L. (2017). Amamentação: validação de tecnologia assistiva em áudio para pessoa com deficiência visual. Acta Paulista de Enfermagem, 30, 122-128, https://www.scielo.br/pdf/ape/v30n2/1982-0194-ape-30-02-0122.pdf.

Oliveira, P. M. P., \& Pagliuca, L. M. F. (2013). Avaliação de tecnologia educativa na modalidade literatura de cordel sobre amamentação. Rev Esc Enfermagem, 47(1):201-208, https://www.redalyc.org/pdf/3610/361033324026_2.pdf.

Oliveira, P. M. P., Rebouças, C. B. D. A., \& Pagliuca, L. M. F. (2009). Construção de uma tecnologia assistiva para validação entre cegos: enfoque na amamentação. Revista Brasileira de Enfermagem, 62, 837-843, https://www.redalyc.org/pdf/2670/267019596006.pdf.

Oriá, M. O. B., Dodou, H. D., Chaves, A. F. L., Santos, L. M. D. A. D., Ximenes, L. B., \& Vasconcelos, C. T. M. (2018). Eficácia de intervenções educativas realizadas por telefone para promoção do aleitamento materno: revisão sistemática da literatura. Revista da Escola de Enfermagem da USP, 52. 52:1-12. https://www.scielo.br/pdf/reeusp/v52/1980-220X-reeusp-52-e03333.pdf.

Prates, L. A., Schmalfuss, J. M., \& Lipinski, J. M. (2015). Rede de apoio social de puérperas na prática da amamentação. Escola Anna Nery, 19, 310-315, https://www.scielo.br/pdf/ean/v19n2/1414-8145-ean-19-02-0310.pdf.

Ribeiro, R. P., Martins, J. T., Marziale, M. H. P., \& Robazzi, M. L. D. C. C. (2012). O adoecer pelo trabalho na enfermagem: uma revisão integrativa. Revista da Escola de Enfermagem da USP, 46, 495-504, https://www.scielo.br/pdf/reeusp/v46n2/a31v46n2.pdf.

Robinson, A., Lauckner, C., Davis, M., Hall, J., \& Anderson, A. K. (2019). Facebook support for breastfeeding mothers: A comparison to offline support and associations with breastfeeding outcomes. Digital health, 5, 1-12 https://journals.sagepub.com/doi/pdf/10.1177/2055207619853397.

Rodrigues, A. P., Dodt, R. C. M., Oriá, M. O. B., Almeida, P. C. D., Padoin, S. M. D. M., \& Ximenes, L. B. (2018). Promoção da autoeficácia em amamentar por meio de sessão educativa grupal: ensaio clínico randomizado. Texto \& Contexto-Enfermagem, 26, 1-10, https://www.scielo.br/pdf/tce/v26n4/0104-0707tce-26-04-e1220017.pdf.

Silva, N. M. D., Waterkemper, R., Silva, E. F. D., Cordova, F. P., \& Bonilha, A. L. D. L. (2014). Conhecimento de puérperas sobre amamentação exclusiva. Revista Brasileira de Enfermagem, 67, 290-295, https://www.scielo.br/scielo.php?pid=S0034-71672014000200290\&script=sci_arttext. Portuguese.

Silva, N. V. D. N. D., Pontes, C. M., Sousa, N. F. C. D., \& Vasconcelos, M. G. L. D. (2019). Tecnologias em saúde e suas contribuições para a promoção do aleitamento materno: revisão integrativa da literatura. Ciência \& Saúde Coletiva, 24, 589-602, https://www.scielosp.org/pdf/csc/2019.v24n2/589-602/pt.

Zocche, D. A. A, Zanatta, E, Adamy, E. K, Vendruscolo, C, Trindade, L. L. (2020). Protocolo para revisão integrativa: caminho para a busca de evidências. Moriá. 